\title{
Especies de Arcobacter y Campylobacter en aves y mamíferos del sur de Chile ${ }^{\#}$
}

\author{
Arcobacter and Campylobacter species in birds and mammals \\ from Southern Chile \\ H Fernández*, F Vera, M P Villanueva \\ Instituto de Microbiología Clínica, Universidad Austral de Chile, Valdivia. Chile.
}

\section{SUMMARY}

\begin{abstract}
The isolation frequency of Arcobacter and Campylobacter species in birds and mammals of Southern Chile was determined. Five species of the Campylobacter genus [C. jejuni subsp. jejuni (25.3\%), C. jejuni subsp. doylei (1.4\%), C. coli (18\%), C. lari (4.6\%) and C. upsaliensis (3.2\%)] and one of the Arcobacter genus [A. butzleri (17.3\%)] were isolated. Dogs are reported as a new reservoir for C. jejuni subsp. doylei; hens, pelicans and sparrows for C. upsaliensis; sparrows for C. lari and dogs, pelicans and sparrows for A. butzleri.
\end{abstract}

Palabras clave: Campylobacter, Arcobacter, reservorios.

Key words: Campylobacter, Arcobacter, reservoirs.

\section{INTRODUCCION}

La Familia Campylobacteraceae comprende los géneros Campylobacter y Arcobacter, los cuales agrupan bacilos Gram negativos curvos, de carácter zoonótico, con amplia distribución en la naturaleza, reconociendo como reservorio natural a una gran variedad de aves y mamíferos (Vadamme 2000).

Varias especies de Campylobacter han sido reconocidas como patógenos intestinales en el ser humano $(C$. jejuni, C. coli, C. upsaliensis) (Vandamme 2000), mientras que las especies Arcobacter butzleri, A. cryaerophilus y A. skirrowii son consideradas como patógenos entéricos emergentes (Fernández y col 2004, Vandenberg y col, 2004). La transmisión de estas bacterias al ser humano se realiza por vía fecal oral a través del consumo de agua o alimentos de origen animal contaminados, o bien, por contacto con animales reservorios (Wesley y col 2000).

Considerando el carácter zoonótico y la extensa distribución de las especies de Arcobacter y Campylobacter en la naturaleza, como también la necesidad de contar con información acerca de su distribución ecológica, con este trabajo nos hemos propuesto aportar antecedentes en relación a su frecuencia de aislamiento en aves y mamíferos del sur de Chile (X Región), ubicados entre los

Aceptado: 08.08.2006.

\# Financiamiento: Proyectos FONDECYT 1030245 y DID-UACh S-2004-01.

* Fax 5663 293300, Casilla de correos 567, Valdivia, Chile; email: hfernand@uach.cl $39^{\circ} 30^{\prime}$ y $41^{\circ} 30^{\prime}$ latitud sur y los $73^{\circ} 30^{\prime}$ y $72^{\circ} 30^{\prime}$ longitud oeste.

\section{MATERIAL Y METODOS}

Para establecer la frecuencia de aislamiento de especies de la Familia Campylobacteraceae se hizo un estudio de prevalencia durante un período de seis meses (abrilseptiembre 2005). Fueron analizadas 314 muestras fecales obtenidas de perro (60), bovino (28), pelícano (60), gorrión (60), gallina (60), pato (25) y pavo (21). Las especies de animales muestreadas fueron seleccionadas por los antecedentes epidemiológicos descritos en la literatura (Fernández y col 1994, Lastovica y Skirrow 2000, Fernández y col 2004) y su número correspondió a aquellos ejemplares que fue posible muestrear durante el período de estudio.

Para el aislamiento de las especies termotolerantes de Campylobacter (C. jejuni subsp. jejuni, $C$. coli y $C$. lari), las muestras fueron sembradas en Agar Skirrow modificado e incubadas a $42{ }^{\circ} \mathrm{C}$ por $48 \mathrm{~h}$ en atmósfera de microaerofilia (Fernández y col 1994). Para aislar las especies no termotolerantes (C. upsaliensis, $C$. jejuni supsp. doylei), se utilizó el método de filtración pasiva sobre agar sangre que consiste en depositar sobre el agar con filtro Millipore estéril de $0,45 \mu \mathrm{m}$ de diámetro y sobre el cual se inoculan $200 \mu \mathrm{L}$ de la muestra suspendida en caldo Brucella. Una vez que la suspensión ha traspasado el filtro, éste se retira y las placas son incubadas por $72 \mathrm{~h}$ a $37{ }^{\circ} \mathrm{C}$, en microaerofilia (Fernández y col 2003).

El aislamiento de las especies de Arcobacter fue realizado utilizando el medio de enriquecimiento de Boer y 
col (1996) incubado durante $48 \mathrm{~h}$ a $26^{\circ} \mathrm{C}$, en aerobiosis con posterior resiembra al medio sólido de Atabay y Corry (1998), el cual fue incubado bajo las mismas condiciones que el medio de enriquecimiento.

La identificación de las especies de Campylobacter fue realizada fenotípicamente utilizando el sistema APICampy ${ }^{\circledR}$ y las de Arcobacter, mediante el método de PCR propuesto por Houf y col (2000).

\section{RESULTADOS Y DISCUSION}

Los resultados obtenidos se muestran en el cuadro 1, observándose que fueron aisladas cinco especies del género Campylobacter (C. jejuni subsp. jejuni, C. jejuni subsp. doylei, C. coli, C. lari y C. upsaliensis) y una del género Arcobacter (A. butzleri). Las especies más frecuentemente aisladas fueron $C$. jejuni subsp. jejuni $(25,3 \%)$, C. coli $(18 \%)$ y A. butzleri $(17,3 \%)$, las cuales fueron encontradas en todas las especies animales estudiadas.

C. jejuni subsp. jejuni y C. coli son reconocidos como los primeros agentes de diarrea en países industrializados y el segundo o tercero en aquellos en vías de desarrollo (Fernández y col 2005). El hecho que hayan sido aislados en todas las especies animales estudiadas no sólo confirma el carácter zoonótico de estas bacterias sino que también la importancia de éstas como reservorios y eventuales fuentes de infección para el ser humano. El hecho de haber sido aisladas de materia fecal de aves para consumo humano pone, también, de manifiesto la importancia de estas aves como fuente de contaminación para alimentos de origen aviar (carne y menudencias), considerados importantes vehículos de estas bacterias
(Lastovica y Kirrow 2000, Vashin y Stoyanchev 2004, Fernández y col 2005).

A. butzleri es considerado como un patógeno emergente, también de carácter zoonótico e involucrado en infecciones entéricas de origen alimentario (Lehner y col 2005). En nuestro estudio, A. butzleri fue aislado de todas las especies animales estudiadas, confirmando su carácter zoonótico y dejando en evidencia el amplio espectro de reservorios que puede colonizar. Por otro lado, fue aislado del tracto intestinal de perros, pelícanos y gorriones, animales aún no descritos como reservorios de esta bacteria (Lehner y col 2005). Los pelícanos pueden constituir un reservorio acuático de estas bacterias $\mathrm{y}$, a la vez, fuente de contaminación para cuerpos de agua y, por extensión, para mariscos filtradores (Fernández y col 2001). Los gorriones, como aves de vida libre, podrían ser fuente de contaminación ambiental e indirecta para el hombre, como fuera sugerido previamente por Fernández y col (2004) a raíz de dos casos de diarrea crónica donde existía evidencia de contacto previo con materia fecal de estas aves. Contrastando con lo descrito en la literatura, llama la atención su relativo alto porcentaje de aislamiento a partir de muestras fecales de gallina (20\%), lo cual podría constituir una diferencia epidemiológica ligada a la distribución ambiental de A. butzleri en nuestra región donde, por los resultados obtenidos en este trabajo y por experiencias locales previas (Fernández y col 2001), se demuestra es más frecuente.

C. lari es un enteropatógeno de baja frecuencia de presentación en seres humanos y ha sido aislado de aves, mamíferos y medio ambiente (Lastovica y Skirrow 2000). En este trabajo, C. lari fue aislado con una fre-

Cuadro 1. Frecuencia de aislamiento de especies de Arcobacter y Campylobacter en aves y mamíferos del sur de Chile. Isolation frequency of Arcobacter and Campylobacter species in birds and mammals from Southern Chile.

\begin{tabular}{|c|c|c|c|c|c|c|c|c|c|c|c|c|c|c|}
\hline \multirow{2}{*}{$\begin{array}{c}\text { Origen / } \\
\mathrm{N}^{\circ} \text { muestras } \\
\text { estudiadas }\end{array}$} & \multicolumn{2}{|c|}{$\begin{array}{l}\text { Muestras } \\
\text { positivas }\end{array}$} & \multicolumn{2}{|c|}{$\begin{array}{c}\text { C. jejuni } \\
\text { subsp. jejuni }\end{array}$} & \multicolumn{2}{|c|}{$\begin{array}{c}\text { C. jejuni } \\
\text { subsp. doylei }\end{array}$} & \multicolumn{2}{|c|}{$\begin{array}{c}\text { C. } \\
\text { coli }\end{array}$} & \multicolumn{2}{|c|}{$\begin{array}{l}\text { C. } \\
\text { lari }\end{array}$} & \multicolumn{2}{|c|}{$\begin{array}{c}C . \\
\text { upsaliensis }\end{array}$} & \multicolumn{2}{|c|}{$\begin{array}{c}\text { A. } \\
\text { butzleri }\end{array}$} \\
\hline & $\mathrm{N}^{\circ}$ & $\%$ & $\mathrm{~N}^{\circ}$ & $\%$ & $\mathrm{~N}^{\circ}$ & $\%$ & $\mathrm{~N}^{\mathrm{o}}$ & $\%$ & $\mathrm{~N}^{\mathrm{o}}$ & $\%$ & $\mathrm{~N}^{\mathrm{o}}$ & $\%$ & $\mathrm{~N}^{\mathrm{o}}$ & $\%$ \\
\hline Perro / 60 & 35 & 58,3 & 12 & 20,0 & 4 & 6,7 & 12 & 20,0 & 1 & 1,7 & 4 & 6,7 & 2 & 3,3 \\
\hline Bovino / 28 & 11 & 39,3 & 3 & 10,7 & 0 & 0 & 1 & 3,6 & 0 & 0 & 0 & 0 & 7 & 25,0 \\
\hline Pelícano / 60 & 45 & 75,0 & 18 & 30,0 & 0 & 0 & 8 & 13,3 & 9 & 15,0 & 2 & 3,3 & 8 & 13,3 \\
\hline Gorrión / 60 & 27 & 45,0 & 10 & 16,7 & 0 & 0 & 8 & 13,3 & 3 & 5,0 & 2 & 3,3 & 4 & 6,7 \\
\hline Gallina / 60 & 40 & 66,7 & 16 & 26,7 & 0 & 0 & 11 & 18,3 & 0 & 0 & 1 & 1,7 & 12 & 20,0 \\
\hline$/ 25$ & 25 & 100 & 10 & 40,0 & 0 & 0 & 5 & 20,0 & 0 & 0 & 0 & 0 & 10 & 40,0 \\
\hline / 21 & 15 & 71,4 & 3 & 14,3 & 0 & 0 & 6 & 28,6 & 0 & 0 & 0 & 0 & 6 & 28,6 \\
\hline Total /314 & 198 & 69,7 & 72 & 25,3 & 4 & 1,4 & 51 & 18,0 & 13 & 4,6 & 9 & 3,2 & 49 & 17,3 \\
\hline
\end{tabular}

\footnotetext{
${ }^{\circledR}$ bioMérieux. Marcy/Etoile, Francia.
} 
cuencia del 4,6\% a partir de tres especies animales, una de las cuales (gorrión) no había sido descrita previamente como reservorio de esta bacteria (Lastovica y Skirrow 2000).

Para C. upsaliensis, agente zoonótico de infecciones intestinales y extraintestinales en el ser humano, se ha descrito como reservorios al perro, gato, pato y monos (Lastovica y Skirrow 2000). C. upsaliensis fue aislado en nuestro estudio a partir de perros y de tres tipos de aves (pelícano, gorrión y gallina), acerca de las cuales no existen registros previos como reservorios de esta especie microbiana.

La especie de menor aislamiento $(1,4 \%)$ fue $C$. jejuni subsp. doylei, siendo encontrada únicamente en perros. Hasta ahora, sólo había sido aislada de gallinas (Vashin y Stoyanchev 2004) y del ser humano, en el cual se presenta en baja frecuencia y con carácter de emergente (Lastovica y Skirrow 2000, Taylor y col 1991, Fernández y col 2003).

La utilización del método de filtración pasiva sobre agar sangre permitió el aislamiento de especies no termofílicas clásicas como C. upsaliensis y C. jejuni subsp. doylei, cuya presencia no es investigada rutinariamente como es el caso de las especies termofílicas clásicas (C. jejuni subsp. jejuni, C. coli y C. lari). Con ello se ha podido ampliar el conocimiento acerca de los animales reservorios de estas bacterias como también el de A. butzleri al emplear métodos específicos para su aislamiento. No obstante ello, pensamos que es necesario estudiar la distribución ambiental de éstas y otras especies de la familia Campylobacteraceae para aportar mayores antecedentes que permitan aclarar y comprender mejor la epidemiología de este grupo bacteriano.

\section{RESUMEN}

Fue determinada la frecuencia de aislamiento de especies de Arcobacter y Campylobacter en aves y mamíferos del sur de Chile. Fueron aisladas cinco especies del género Campylobacter [C. jejuni subsp. jejuni (25,3\%), C. jejuni subsp. doylei (1,4\%), C. coli (18\%), C. lari $(4,6 \%)$ y C. upsaliensis $(3,2 \%)]$ y una del género Arcobacter [A. butzleri (17,3\%)]. Se describe al perro como nuevo reservorio para C. jejuni subsp. doylei, a gallina, pelícano y gorrión para C. upsaliensis, al gorrión para C. lari y al perro, pelícano y gorrión para A. butzleri.

\section{REFERENCIAS}

Atabay HI, J Corry. 1998. Evaluation of a new Arcobacter enrichment medium and comparison with two media developed for enrichment of Campylobacter spp. Int J Food Microbiol 41, 53-58.

De Boer E, JJ Tilburg, DL Woodward, H Lior, WM Johnson. 1996. A selective medium for the isolation of Arcobacter from meats. Lett Appl Microbiol 23, 64-66.

Fernández H, K Kahler, R Salazar, M Ríos. 1994. Prevalence of thermotolerant species of Campylobacter and their biotypes in children and domestic birds in Southern Chile. Rev Inst Med Trop 36, 433-436.

Fernández H, L Otth, M Wilson, R Rodríguez, B Proboste, C Saldivia, P Barría. 2001. Occurrence of Arcobacter sp. in river water, mussels and chicken livers in Southern Chile. Int J Med Microbiol, 291, Suppl 31, 140.

Fernández H, R Rodríguez, C Barudi, M Lobos. 2003. A case of acute diarrhea due to the emerging pathogen Campylobacter jejuni subsp. doylei in Southern Chile. Braz. J Microbiol 34, 52-54.

Fernández H, S Krause, MP Villanueva. 2004. Arcobacter butzleri an emerging enteropathogen: communication of two cases with chronic diarrea. Braz J Microbiol 35, 216-218.

Fernández H, A García, MP Villanueva. 2005. Serotipos de Campylobacter jejuni ssp. jejuni en carne de ave para consumo humano y en muestras de heces de niños con diarrea. Arch Med Vet 37, 79-81.

Houf K, A Tutenel, L de Zutter, J van Huf, P Vandamme. 2000. Development of a multiplex PCR assay for the simultaneous detection of Arcobacter butzleri, Arcobater cryaerophilus and Arcobacter skirrowii. FEMS Microbiol Lett 193, 89-94.

Lastovica A, M Skirrow. 2000. Clinical significance of Campylobacter and relatd species other than Campylobacter jejuni and C. coli. En: Nachamkin I, M Blaser (eds). Campylobacter $2^{\text {nd }}$ Edition. ASM Press, Washington DC, USA, Pp 89-120.

Lehner A, T Tasara, R Stephan. 2005. Relevant aspects of Arcobacter spp. as potential foodborne pathogen. Int J Food Microbiol 102, 127-135.

Taylor DN, JA Kiehlbauch, W Tee, C Pitarangsi, P Echeverría. 1991. Isolation of group 2 aerotolerant species from Thai children with diarrhea. J Infect Dis 163, 1062-1067.

Vandamme P. 2000. Taxonomy of the Family Campylobacteraceae. En: Nachamkin I, Blaser MJ (eds). Campylobacter 2nd Edition. ASM Press, Washington, DC, USA, Pp 3-26.

Vandenberg O, A Dediste, K Houf, S Ibekwem, H Souayah, S Cadranel, N Douat, G Zissis, JP Butzler, P Vandamme. 2004. Arcobacter species in humans. Emerg Infect Dis 10, 1863-1867.

Vashin IT, TT Stoyanchev. 2004. Incidence and microbial diversity of Campylobacter spp. isolates during the slaughterhouse processing of poultry and critical control points of the process. Bulg J Vet Med 7, 173-180.

Wesley IV, SJ Wells, KM Harmon, A Green, L Schroeder-Tucker, G Glover, I Suddique. 2000. Fecal shedding of Campylobacter and Arcobacter spp. in dairy cattle. Appl Environ Microbiol 66, 1994-2000. 